\title{
Simulated body fluid and water absorption effects on poly(methyl methacrylate)/hydroxyapatite denture base composites
}

\author{
W. L. Tham, W. S. Chow*, Z. A. Mohd Ishak \\ School of Materials and Mineral Resources Engineering, Engineering Campus, Universiti Sains Malaysia, Nibong Tebal, \\ 14300 Penang, Malaysia
}

Received 4 March 2010; accepted in revised form 13 May 2010

\begin{abstract}
Poly(methyl methacrylate) (PMMA)/hydroxyapatite (HA) composite has potential application in denture base materials. The denture base materials should exhibit good mechanical properties and dimensional stability in moist environment. Silane coupling agent [3-methacryloxypropyltrimethoxy silane $(\gamma$-MPS)] was used to treat the HA fillers in order to enhance the interfacial interaction between the PMMA and HA. In this research, the kinetics and effects of Simulated Body Fluid (SBF) and water absorption on the flexural properties of PMMA/HA composites were studied for an immersion duration of 2 months. The mathematical treatment used in analyzing the data was the single free phase model of diffusion, which assumed Fickian diffusion and utilized Fick's second law of diffusion. The kinetics of water absorption of the PMMA/HA composites conformed to Fickian law behavior, whereby the initial moisture absorption follows a linear relationship between the percentage gain at any time $t$ and $t^{1 / 2}$, followed by saturation. It was found that the equilibrium moisture content and the diffusion coefficient are depending on the concentration of $\gamma$-MPS in PMMA/5HA composites. The reduction of equilibrium moisture content of PMMA/5HA is due to the hydrophobic behavior of $\gamma$-MPS and compatibility of PMMA with HA. The retention ability in flexural modulus and strength of PMMA/HA composites upon subjected to water absorption are considerably good. The reduction of flexural strength of the PMMA/HA composites after water absorption and SBF absorption could be attributed to the plasticizing effect of water molecules.
\end{abstract}

Keywords: polymer composites, poly(methyl methacrylate), hydroxyapatite, water absorption, mechanical properties

\section{Introduction}

Poly(methyl methacrylate) (PMMA) has been widely used as a biomaterial in dentistry, orthopedic retainers, bone replacement, and eyeballs [1]. PMMA continues to be used as denture base materials because of its favorable characteristics, ease of processing, stability in the oral environment, ease in repair and superior esthetics [2-3]. However, the primary problem of PMMA is its poor strength characteristics, low impact strength and fatigue resistance [4]. Thus, there is a need to improve the performance of PMMA in denture base application. Several methods have been used to modify the properties of PMMA denture base materials. Approaches to strengthening the acrylic resin prosthesis have included modifying or reinforcing the resin by using filler and fiber, and graft copolymerization with high-impact resins [2-3]. Some effort has been made to increase the impact strength by the incorporation of a rubber phase in the PMMA, however, such materials have compromised flexural properties [5]. Hydroxyapatite (HA) $\left[\mathrm{Ca}_{10}\left(\mathrm{PO}_{4}\right)_{6}(\mathrm{OH})_{2}\right]$ was used in various biomedical fields such as dental material, bone substitute and hard tissue paste [6]. Hydroxyapatite (HA)-reinforced polymers have many potential clinical appli-

*Corresponding author, e-mail: shyang@eng.usm.my

(c) BME-PT 
cations, e.g. bone cement, dental implants, coating of joint replacement prosthesis etc. [7]. The mechanical properties of PMMA/HA composites could be limited by the incompatibility between the PMMA and HA. Thus modification of PMMA/HA composites is required in order to achieve a high performance denture base materials with better mechanical properties. Polymeric compatibilizer and coupling agent can play a role to improve the interaction and adhesion between the organic PMMA matrix and inorganic HA particles. From our previous work, it was found that the silane coupling agent [3-methacryloxypropyltrimethoxy silane $(\gamma$-MPS)] can improve the mechanical and thermal properties of PMMA/HA significantly owing to the better adhesion.

The use of heat-polymerized, permanent, acrylic resin denture bases has certain advantages owing to their strong and rigid behavior, in which, they should provide the retention and stability of the final denture [8]. Water absorption of PMMA/HA composite is an important study for dentistry science. In oral environment, dental restorative materials are exposed to saliva containing water. Sometimes, the dental materials are exposed to exogenous substances such as acids, base, salts, alcohols, oxygen, during drinking or eating. Water absorption caused plasticization, lowering of the glass transition temperature $\left(T_{g}\right)$ and may affect the mechanical properties of the polymeric denture based materials. Hydrolytic degradation of the hygroscopic filler may occur when subjected to water absorption [9]. Excessive water uptake may lead to breakdown of the polymer itself, debonding of filler from matrix substance, chemical composition changes by leaching and lead to the ingress of microorganism [10]. Besides, precipitation and swelling phenomena may produce voids and cracks, leaching the interface results in the loss of strength due to corrosion. All of these processes may lead to nucleation and growth of microcracks [11]. Moreover, due to the low molecular weights and high solubility of residual unreacted monomer such as triethylenegylcol-dimethacrylate (TEGDMA) and 2-hydroxyethyl methacrylate (HEMA), may be released from swollen dental adhesives through dental tubules and elicit adverse biological reactions. Dissolution of components from the adhesive material itself can have a poten- tial impact on its structural stability and consequently expedite the degradation of the resin-dentin bonds [12]. The uptake of water by an unfilled resin is a free volume and thermodynamics-controlled process [9]. A large number of air voids was observed in the posterior composites and this probably leads to high water absorption. Air voids incorporated in the materials may result in higher water solubility. The presence of polar and hydrophilic functional groups in acrylic resins may increase their polarity and facilitate uptake of bound water to form primary and secondary hydration shells around the polar groups via hydrogen bonding [13].

Simulated Body Fluid (SBF) test, a method that is well recognized to characterize the in vitro bioactivity of ceramic materials, consists in their immersion in an aqueous SBF solution which simulates the properties of human plasma for certain period and verifies the formation of the hydroxyapatite (HA) layer on the surface of the samples [14]. The quantity of water molecules and SBF absorbed by the resin matrix of dental resin based composite and the rate of water sorption have been identified as being diffusion controlled. The kinetics of water absorption of the resin based composites conformed to Fickian law behavior in general agreement with the plots of percentage of water uptake [\%] against any time $t^{1 / 2}$ remained linear in the initial stages of water absorption followed by a decrease in the rate of diffusion [15]. Theoretically, the water sorption process is determined by the diffusion coefficient and the boundary conditions at the surface of the material. It is an estimation method of the diffusion coefficient using the solution of Fick's second law [16]. Denture base acrylic resins are subjected to many various types of intraoral and extraoral stresses and water contact, e.g. immersion in water-based solution for cleaning purpose, contact with drinking water (hot and cold) and etc. The mechanical properties of PMMA/HA denture base materials during their service life is important. This paper reports on the effects of water absorption and SBF on the properties of PMMA/HA composites with and without surface treatment by silane coupling agent. The maximum moisture absorption $\left(M_{m}\right)$ and diffusion coefficient $(D)$ of PMMA/5HA composites upon subjected to water absorption and SBF will be determined. 


\section{Materials and methods}

\subsection{Materials}

The solid components consist of PMMA with typical molecular weight of 996000 (Product no: 182265, Sigma Aldrich, USA) and $0.5 \%$ benzoyl peroxide (BPO) (Product no: 801641, Merck Chemical, Germany). The monomers used consist of methyl methacrylate (MMA) (Product no: M 55909, Sigma Aldrich, USA) stabilized with 10-100 ppm monomethyl ether hydroquinone as inhibitor and ethylene glycol dimethacrylate (EGDMA) (Product no: 335681, Sigma Aldrich, USA). Hydroxyapatite (HA) in powder forms was supplied by Sigma Aldrich (USA) (Product no: 289396) with an average specific surface area (BET) $50 \mathrm{~m}^{2} / \mathrm{g}$ and average particle diameter of $18 \mu \mathrm{m}$. The $\gamma$-MPS was supplied by Sigma Aldrich (USA) with 98\% purified contents.

\subsection{Silanization of hydroxyapatite particles}

The HA filler was silanized using 3-methacryloxypropyltrimethoxy silane ( $\gamma$-MPS) in 90\% methanol and $10 \%$ distilled water. The $\gamma$-MPS was diluted in water-methanol solution, with $\mathrm{pH}$ of 4 to 5 , which adjusted with acetic acid. The Equation (1) gives the amount of required silane to obtain minimum uniform coverage of the filler particles $(X)$ [17-18]:

$$
X=\left(\frac{A}{w}\right) f
$$

where $A$ is the surface area of the HA filler $\left(50 \mathrm{~m}^{2} / \mathrm{g}\right)$ and $f$ is the amount of HA. It was found that each silane molecule covers $1.11 \mathrm{~nm}^{2}$ of filler and so that the surface area coverage per gram of MPS is $w=2525 \mathrm{~m}^{2} \cdot \mathrm{g}^{-1}$. According to Söderholm and Shang [19], each MPS molecule occupied at least $1.11 \mathrm{~nm}^{2}$ for pyrogenic colloidal silica (CabO-Sil). Karabela and Sideridou [17] reported that for the Aerosil silica nanoparticles (with the specific surface area (BET) in the range of 35$65 \mathrm{~m}^{2} / \mathrm{g}$ ), the silane molecule covers $1.11 \mathrm{~nm}^{2}$ of filler and, thus the surface area coverage per gram of MPS is $w=2525 \mathrm{~m}^{2} \cdot \mathrm{g}^{-1}$. Four different concentrations of $\gamma$-MPS (i.e. 2, 4, 6 and $8 \%$ based on the weight of HA filler) were use to modify HA. Figure 1 shows the possible silanization mechanism of HA by $\gamma$-MPS, and the interaction between PMMA, HA and $\gamma$-MPS.

\subsection{Preparation of PMMA/5HA composites}

The powder component was prepared by mixing of PMMA, 5\% HA and $0.5 \%$ BPO. The liquid component was prepared by mixing MMA monomer and EGDMA with a predetermined ratio of $9: 1$. The ratio of powder to liquid was set at 10:4 according

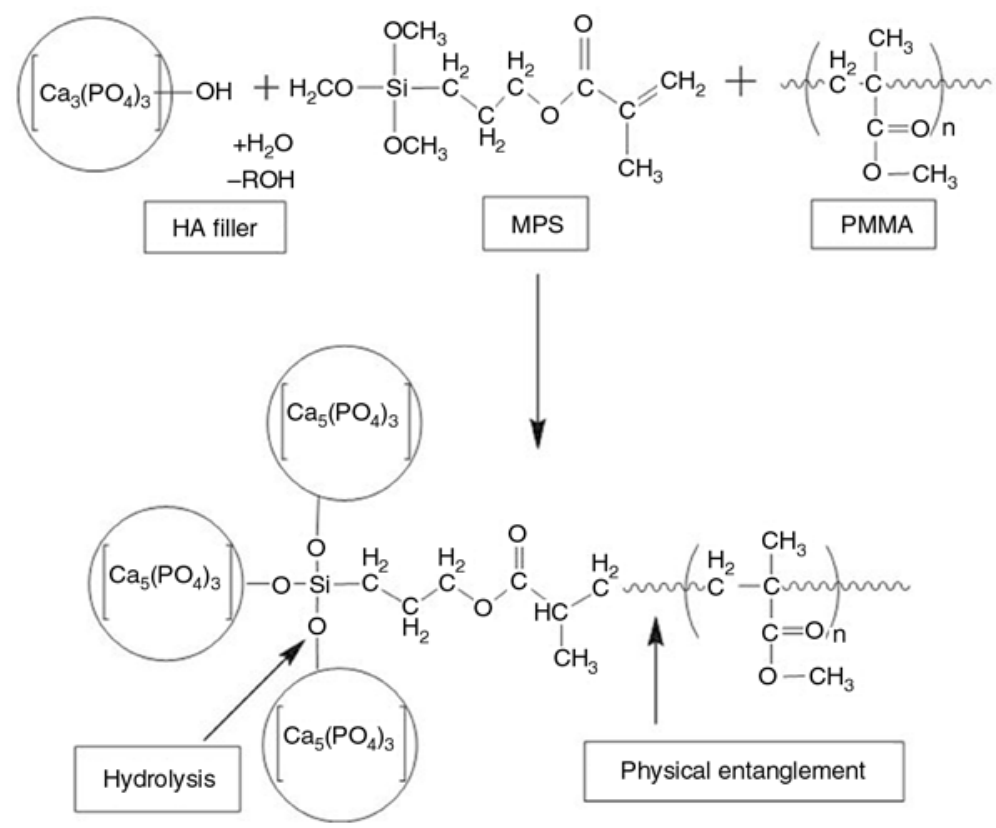

Figure 1. Possible interaction of $\gamma$-MPS, HA and PMMA 
to the dental laboratory practice. The powder and liquid components were mixed together. After achieving the dough stage, the mixture was packed into a mold with the pressure of $14 \mathrm{MPa}$ using compressor at room temperature for $25 \mathrm{~min}$. The polymerization was carried using a water bath at $78^{\circ} \mathrm{C}$ and $90 \mathrm{~min}$.

\subsection{Characterization of PMMA/5HA composites}

\subsubsection{Flexural test}

Three-point bending tests were performed according to ASTM D790 using an Instron 3366 machine (USA). The support span length was set at $50 \mathrm{~mm}$. The crosshead speed was set at $2 \mathrm{~mm} / \mathrm{min}$. At least five samples for each formulation were examined. The flexural modulus and flexural strength were recorded.

\subsubsection{Water absorption}

The water absorption tests were conducted according to ASTM D 570-98. Specimens as flexural bars were dried in a vacuum oven at $70^{\circ} \mathrm{C}$ until a constant weight was attained. Specimens (i.e. PMMA, PMMA/5HA composites and PMMA/ $\gamma$-MPS treated 5HA composites) were placed in a container of distilled water at temperature of $27^{\circ} \mathrm{C}$. The weight gain of the specimens was measured as a function of time after removing the water on their surfaces. The percentage gain at time $t,\left(M_{t}\right)$ as a result of moisture absorption was determined by Equation (2):

$$
M_{t}=\frac{W_{w}-W_{d}}{W_{d}} \cdot 100 \%
$$

where $W_{d}$ and $W_{w}$ denote the initial weight of specimen prior to exposure to the water absorption and weight of specimen after exposure to water absorption, respectively.

$$
\frac{M_{t}}{M_{m}}=1-\frac{8}{\pi^{2}} \exp \left[-\left(\frac{D t}{h^{2}}\right) \pi^{2}\right]
$$

For Equation (3), the maximum moisture absorption $\left(M_{m}\right)$ was calculated as an average value of several consecutive measurements that showed no appreciable additional absorption, $h$ is the thickness of the specimens and $D$ is the diffusion coefficient. The diffusion coefficient was calculated using Equation (4):

$$
D=\frac{\pi h^{2}\left(M_{2}-M_{1}\right)^{2}}{16 M_{m}^{2}\left(t_{2}^{1 / 2}-t_{1}^{1 / 2}\right)}
$$

\subsubsection{Simulated Body Fluid (SBF) absorption}

SBF absorption test was carried out according to ISO specification 1567-2001. The specimens (in flexural bars geometry) were dried in a vacuum oven at $70^{\circ} \mathrm{C}$ until a constant weight was attained. The specimens were then placed in a container of SBF. The containers were then put in water bath at temperature of $37^{\circ} \mathrm{C}$. The SBF was supplied by B-Braun Medical Industries (Penang, Malaysia). The weight gain of the specimens was measured as a function of time after removing the water on their surfaces. The percentage gain at time $t,\left(M_{t}\right)$ as a result of SBF absorption was determined by Equation (2) and diffusion coefficient was calculated using Equation (4).

\subsubsection{Field Emission Scanning Electron Microscopy (FESEM)}

The morphological studies of flexural bar samples were carried out on FESEM Supra 35VP (Carl Zeiss, Germany) equipped with the Gemini column. The acceleration voltage used was $5 \mathrm{kV}$. The specimens' surface was coated by Aurum/Palladium alloy in order to prevent electrostatic discharge.

\subsection{Statistical analysis}

The flexural test data reported in the Table 2 and 3 represent the mean values and standard deviation of replicates. The data were statistically analyzed with a one-way analysis of variance (ANOVA) to determine significant differences was used at a significant level set at $\mathrm{p} \leq 0.05$, for analysis of the results. It was followed by the univariate analysis of variance to identify the tests of between-subjects effects. 


\section{Results and discussions}

\subsection{Kinetics of water absorption}

Figure 2 shows the effect of $\gamma$-MPS concentration on the water uptake of PMMA/5HA composites during water absorption at room temperature. It can be seen that the percentage of water uptake of the PMMA/5HA composites is higher than that of PMMA and PMMA/ $\gamma$-MPS treated 5HA composites. The maximum moisture absorption $\left(M_{m}\right)$ was reached after 2 months of water absorption. The water sorption, water solubility and associated diffusion coefficient of dental resin-based composite materials are dependent on the chemistry of the monomer resins and the extent of polymerization of the polymer matrix, the present of solubility impurities, filler particles size, filler distribution, and the interfacial properties between filler with resin matrix [10, 15, 20]. According to Harper et al. [21], the weight change of the specimens was the combination of increase in weight due to water absorption and loss in weight caused by leaching of the monomer. Table 1 shows the percentage of maximum moisture absorption $\left(M_{m}\right)$ and diffusion coefficient $(D)$ of PMMA, PMMA/5HA composites and PMMA $/ \gamma$-MPS treated 5HA composites. The $M_{m}$ of PMMA/5HA composites is approximately $2.12 \%$, while the $M_{m}$ of PMMA is about $1.71 \%$. The notable weight gain of PMMA/5HA compos-

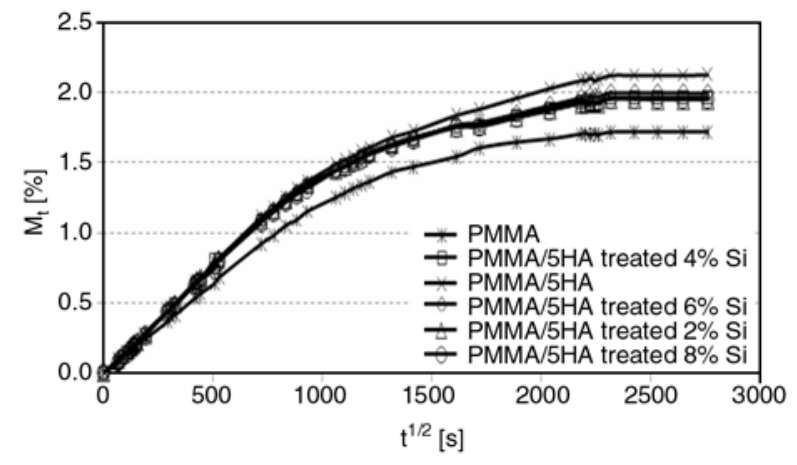

Figure 2. The effects of $\gamma$-MPS concentration on the water uptake of PMMA upon subjected to water absorption ites can be attributed to the presence of the HA filler. The moisture absorption of HA is due to the free and reactive hydroxyl groups on the HA particles which exhibit good affinity to water molecules. Besides, there are micro-voids in between PMMA and HA caused by the difference in the chemical nature of PMMA and HA. This phenomenon is attributed to poorly bonded area and weak interfacial adhesion between the hydrophilic HA filler and the hydrophobic PMMA. Therefore, water molecules could penetrate into these micro-voids or accumulated at the filler-matrix interface. A similar phenomenon was observed by Yang et al. [22] in LDPE composites containing 30\% lignocellulosic filler. Excessive water uptake can promote breakdown causing a filler-matrix debonding in the presence of porosity and inclusions of filler particle aggregates. This phenomenon would be involved in the water absoprtion as they appear loosely embedded in the matrix. The amount of water can be accumulated at the interface between these agglomerates and the matrix. However, the presence of the $\gamma$-MPS introduced a hydrophobic layer on HA particle surfaces, thus the percentage of water uptake was slightly decreased compared to PMMA/5HA composites. According to Santos et al. [23], during the silanazation process, multiple layers of silane form a film around the filler particles, which is either physically or chemically attached to the filler particles, and thus limits the water absorption.

Fickian diffusion and Fick's second law of diffusion was used to determine the diffusivity parameter. From Table 1, it can be seen that the $M_{m}$ of PMMA/ $\gamma$-MPS treated 5HA composite is lower than that of PMMA/5HA composites. Although all the PMMA/5HA composites (with and without silane coupling agents) shows $M_{m}$ value higher than pure PMMA. However, it is interesting to note that the diffusion coefficient $(D)$ of PMMA/5HA composites is lower than that of PMMA. The mecha-

Table 1. Maximum moisture content $\left(M_{m}\right)$ and diffusion coefficient $(D)$ of PMMA, PMMA/5HA composites and PMMA/ $\gamma$-MPS treated 5HA composites after being subjected to water absorption and SBF tests

\begin{tabular}{|c|c|c|c|c|}
\hline \multirow{2}{*}{ Material } & \multicolumn{2}{|c|}{$\mathbf{M}_{\mathrm{m}}[\%]$} & \multicolumn{2}{|c|}{$\mathrm{D}\left[\mathrm{m}^{2} / \mathrm{s}\right]$} \\
\hline & Water absorption & SBF absorption & Water absorption & SBF absorption \\
\hline PMMA & 1.71 & 1.96 & $1.25 \cdot 10^{-12}$ & $1.64 \cdot 10^{-12}$ \\
\hline PMMA/5HA & 2.12 & 2.36 & $1.07 \cdot 10^{-12}$ & $1.94 \cdot 10^{-12}$ \\
\hline PMMA/5HA treated $2 \% \mathrm{Si}$ & 1.95 & 2.02 & $1.04 \cdot 10^{-12}$ & $1.95 \cdot 10^{-12}$ \\
\hline PMMA/5HA treated $4 \% \mathrm{Si}$ & 1.96 & 2.06 & $1.01 \cdot 10^{-12}$ & $1.91 \cdot 10^{-12}$ \\
\hline PMMA/5HA treated $6 \% \mathrm{Si}$ & 2.00 & 2.14 & $1.03 \cdot 10^{-12}$ & $1.82 \cdot 10^{-12}$ \\
\hline PMMA/5HA treated $8 \% \mathrm{Si}$ & 1.95 & 2.06 & $1.00 \cdot 10^{-12}$ & $1.83 \cdot 10^{-12}$ \\
\hline
\end{tabular}


nism through which water diffuses into polymeric materials can be explained as either infiltration into the free space (i.e. micro-voids and other morphological defects) or specific molecular interaction being controlled by the available hydrogen bond at hydrophilic sites [24]. According to Tang et al. [25], this may be related to the barrier contribution of filler inclusions to water transportation. Accordingly, when the transportation paths of the water molecules and the water interaction within the composites increase, it will reduce the diffusion coeficient of the composites. The slightly decrease of the diffusion coefficient for PMMA/ $\gamma$-MPS treated 5HA composites compared to PMMA/5HA composites may suggest the reduction of microvoids for water accumulation and a hydrophobic behavior due to better interaction of HA filler and PMMA matrix by silanization of HA.

\subsection{Effects of water absorption on the flexural properties of PMMA composites}

Table 2 shows the flexural properties of the PMMA, its composites in control, wet and redried states after being subjected to water absorption for 1 and 2 months. It can be seen that the flexural modulus of PMMA/ $\gamma$-MPS treated HA composites decreased slightly after being subjected to water absorption for 1 month. There is no further significant reduction of the flexural modulus of PMMA composites after being subjected for 2 months to water absorption for re-dried samples. Table 2 also shows the percentage of recovery of the flexural modulus for the PMMA composites. The recovery of flexural modulus for PMMA composites is excellent. This can be seen from Table 2 that the flexural modulus of re-dried samples is almost comparable to that of the wet sample. According to Matinlinna and Vallittu [26], sometimes the siloxane films of adequate bonding quality can still be formed since the remaining non-hydrolyzed alkoxy groups are assumed to become hydrolyzed in air and thus activated. When these PMMA/ $\gamma$-MPS treated HA composites are immersed in water, the inorganic hydroxyl surface of HA may further react with the remaining and available hydrolysable alkoxy groups of $\gamma$-MPS. Thus, the increment of flexural modulus and strength for PMMA composites may be associated to the post-chemical reaction during re-drying process. Similar results were reported by Harper et al. [21], the increase in strength of poly(ethyl methacrylate) cement after 12 weeks immersion may be due to water being redistributed at that time, which is caused by post curing. Reis et al. [27] had reported that denture base acrylic resin after water storage for 30 days, there was an increased in flexural strength. This could be explained by continuous polymerization of polymers. Residual monomer may act as a plasticizer and thus, residual monomer leached from the specimens could also contribute to the increase in flexural strength after water storage.

Table 2. Flexural properties of PMMA, PMMA/5HA composites and PMMA/ $\gamma$-MPS treated 5HA composites in wet and re-dried states after being subjected to water absorption for various condition $(n=3)$

\begin{tabular}{|c|c|c|c|c|c|c|}
\hline \multirow{3}{*}{ Material } & \multirow{3}{*}{ Flexural properties } & \multirow{3}{*}{ Control } & \multicolumn{4}{|c|}{ Duration of immersion } \\
\hline & & & \multicolumn{2}{|c|}{1 month } & \multicolumn{2}{|c|}{2 months } \\
\hline & & & Wet & Redried & Wet & Redried \\
\hline \multirow{2}{*}{ PMMA } & Flexural modulus [GPa] & 2.3 & $2.4(103.3)$ & $2.6[113.2]$ & $2.5(110.3)$ & $2.8[122.4]$ \\
\hline & Flexural strength [MPa] & 77.7 & $90.0(115.8)$ & $72.2[92.8]$ & $71.9(92.6)$ & $73.2[94.3]$ \\
\hline \multirow{2}{*}{ PMMA/5HA } & Flexural modulus [GPa] & 2.5 & $2.5(104.4)$ & $2.8[110.1]$ & $2.6(103.5)$ & $3.1[122.1]$ \\
\hline & Flexural strength [MPa] & 50.5 & $57.6(114.0)$ & $57.3[113.6]$ & $45.7(89.4)$ & $63.8[126.4]$ \\
\hline \multirow{2}{*}{ PMMA/5HA treated $2 \% \mathrm{Si}$} & Flexural modulus [GPa] & 2.7 & $2.6(96.3)$ & $2.9[105.5]$ & $2.7(100.0)$ & $3.0[110.28]$ \\
\hline & Flexural strength [MPa] & 56.5 & $56.1(99.3)$ & $63.8[120.8]$ & $49.4(93.2)$ & $55.3[97.9]$ \\
\hline \multirow{2}{*}{ PMMA/5HA treated $4 \% \mathrm{Si}$} & Flexural modulus [GPa] & 2.8 & $2.7(97.4)$ & $2.9[102.8]$ & $2.7(91.7)$ & $3.0[106.8]$ \\
\hline & Flexural strength [MPa] & 61.4 & $56.2(91.5)$ & $58.1[102.8]$ & $47.7(84.3)$ & $61.9[109.4]$ \\
\hline \multirow{2}{*}{ PMMA/5HA treated $6 \% \mathrm{Si}$} & Flexural modulus [GPa] & 2.9 & $2.7(93.7)$ & $2.9[100.4]$ & $2.7(91.7)$ & $3.0[105.3]$ \\
\hline & Flexural strength [MPa] & 61.4 & $55.7(90.8)$ & $54.6[89.0]$ & $47.9(71.3)$ & $60.0[97.9]$ \\
\hline \multirow{2}{*}{ PMMA/5HA treated $8 \% \mathrm{Si}$} & Flexural modulus [GPa] & 2.8 & $2.7(96.5)$ & $2.9[104.9]$ & $2.8(100.4)$ & $3.1[111.5]$ \\
\hline & Flexural strength [MPa] & 52.4 & $51.7(98.6)$ & $57.8[110.4]$ & $52.6(100.4)$ & $63.0[120.3]$ \\
\hline
\end{tabular}

Remark 1: The values in parentheses ( ) are the percentage retention of the flexural properties after water absorption and the values in brackets [ ] are the percentage recovery of the flexural properties after re-drying

Remark 2: Statistically analyzed with one-way ANOVA correspond to various immersion condition indicate significant different $(P<0.05)$ in flexural modulus $\left(R^{2}=0.732\right)$ and flexural strength $\left(R^{2}=0.826\right)$ 
Table 2 shows that the flexural strength of PMMA composites was slightly increased after 1 month of water absorption. After 2 months of water absorption, flexural strength for all specimens was decreased in wet state. Table 2 also shows that the percentage of retention of the flexural strength of all PMMA/5HA composite was decreased after 2 months of water absorption. This is due to the plasticizing effect of water molecules which diffused into the matrix and filler. However, the flexural strength of treated PMMA/5HA composite could recover greatly by considering its properties after 2 months of water absorption in the re-dried state. The moisture diffused predominantly into the matrix resin, resulting in a significant mismatch of the moisture induced volumetric expansion between the matrix and the filler. When the stress exceeds the strength of the interphase region, debonding may take place between the filler and the matrix resulting in an irreversible damage to the dental composite [28]. The flexural strength of the PMMA/5HA composites decreased due to the weak interfacial bonding between PMMA and HA [29]. This will lead to increased micro voids in the composites, and, as a result, increase of water absorption [22]. When HA particles are embedded in the highly entangled polymer chains, there are micro-voids (or free volume voids) in the composite interface and even within the matrix polymer. These micro-spaces are essential for the relaxation of polymer chains and induce transportation of water molecules when soaking in an aqueous environment [25]. Accordingly, the water molecule can acts as internal plasticizer. It is interesting to note that PMMA/8\% $\gamma$-MPS treated 5HA composites still remain of the same strength as the control sample. This indicates that the HA treated with $8 \%$ $\gamma$-MPS can provide better hydrolytic stability without diminishing the flexural properties of the PMMA composites during water absorption.

\subsection{Kinetics of SBF absoprtion}

Figure 3 shows the effect of $\gamma$-MPS concentration on the moisture uptake of PMMA and PMMA/5HA composites during $\mathrm{SBF}$ absorption at $37^{\circ} \mathrm{C}$ up to 2 months of immersion. The initial linear region showed a faster rate of SBF uptake for PMMA/ 5HA composite followed by the PMMA/ $\gamma$-MPS treated 5HA composites and unfilled PMMA. All

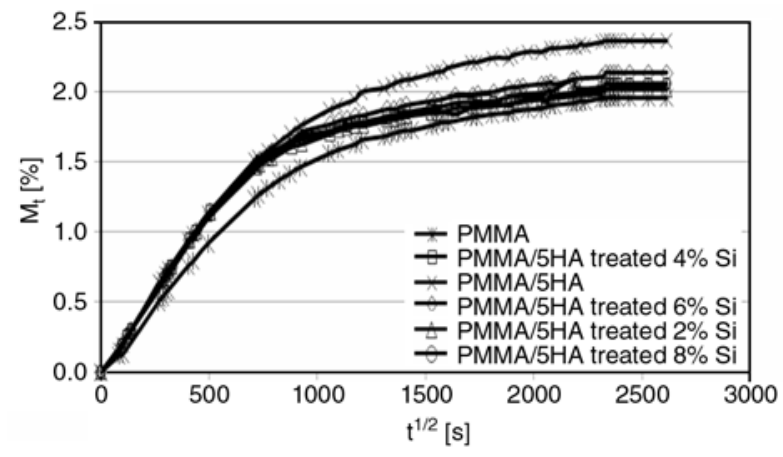

Figure 3. The effects of $\gamma$-MPS concentration on the SBF uptake of PMMA upon subjected to SBF absorption

specimens showed an SBF uptake process involving a rapid Fickian process which is linear to $t^{1 / 2}$ followed by a slower rate of uptake which is indicated as matrix saturation. The SBF and liquid uptake into polymer matrix is governed by either the free volume theory, in which water diffuses through substrates or the interaction theory, in which it is controlled solely by the available hydrogen bond at the polar sites [13]. The equilibrium of SBF absorption $\left(M_{m}\right)$ was achieved after 2 months of immersion. From Table 1, it can be seen that the maximum SBF absorption $\left(M_{m}\right)$ of PMMA and PMMA/5HA composites is approximately $1.96 \%$ and $2.36 \%$, respectively. However, the SBF uptake of PMMA $/ \gamma$-MPS treated 5HA composite was decreased to $2.02-2.14 \%$ as a function of the concentration of $\gamma$-MPS. This suggests that a better bonding between the $\gamma$-MPS treated HA filler and the PMMA. According to Deb et al. [10], the silanization of the mineral phase probably inhibits water uptake due to hydrophobic surface.

Table 1 also shows the diffusion coefficients $(D)$ of PMMA, PMMA/5HA composites and PMMA/ $\gamma$-MPS treated 5HA composites after SBF immersion at $37^{\circ} \mathrm{C}$ for 2 months. The diffusion coefficient (D) of PMMA/5HA composites ( $\left.D=1.94 \cdot 10^{-12} \mathrm{~m}^{2} / \mathrm{s}\right)$ is somewhat higher than that of PMMA $(D=$ $\left.1.64 \cdot 10^{-12} \mathrm{~m}^{2} / \mathrm{s}\right)$. According to Yiu et al. [13], the polarity and hydrophilic sites in the composite may lead to trapping sites to stop the water for interaction which will subsequently hinder the water molecule diffusion. It is interesting to note that the $\gamma$-MPS treated PMMA/5HA composites (6\% $\gamma$-MPS and $8 \% \gamma$-MPS) are slightly lower than untreated PMMA/5HA composite. This is again attributed to the better interfacial between PMMA and HA, which in turn, reduces the transportation path of the 
water molecules in the PMMA matrix. According to Drummond [11], the bonding of glass to resin through silane agents (formation of oxane bonds) other than simple chemical reactivity is good in explained by interdiffusion and interpolymer network formation in the interphase region. According to Karabela and Sideridou [17], as the dental composites are placed in the oral environment absorption of water takes place, which may lead to the degradation of the silane coupling agent and reinforcing filler. The debonded interphase is the fastest path for water migration into the interior layer of resin composite. With the unsilanized composite, degradation is the most severe resulting in the deepest degradation depth. However, as the fillers are silanized, the silane film absorbing water before undergoing hydrolysis resists water diffusion into composite. The diffusion coefficients of untreated and treated PMMA/5HA composite are generally higher than that of PMMA. This may be due to the ions in the SBF, i.e. $\mathrm{Na}^{+}, \mathrm{K}^{+}, \mathrm{Ca}^{2+}, \mathrm{Cl}^{-}$ and $\mathrm{HCO}_{3}^{-}$. The presence of these ions may leads to the formation of apatite on the HA particles (which are distributed on the surface of the sample) and thus these ion concentrations, especially that of $\mathrm{HCO}_{3}^{-}$induce increased rate of diffusivity in the sample by interacting with the hydroxyl groups of the specimen. According to Greish and Brown [30], the mechanism of apatite on the surfaces of bioactive glasses, such as calcium, phosphate, and silicate ions are released from the bioactive glass surfaces, leaving a hydrated silica layer. This layer has been reported to enhance the formation of the apatite nuclei. The dissolution of calcium and phosphate ions increases the the activity of the ionic components of apatite in the surrounding fluid, leading to the precipitation of the apatite nuclei on the hydrated silica layer, which later grow spontaneously. Verestiuc et al. [31] reported that for shorter interaction time of SBF with the substrate, the concentration of $\mathrm{Ca}^{2+}$ ion and $\left(\mathrm{PO}_{4}\right)^{3^{-}}$increases. After few days, the $\mathrm{Ca}^{2+}$ ion and $\left(\mathrm{PO}_{4}\right)^{3^{-}}$variations evolve oppositely due to the diffusion of positive and negative ions having different motilities in SBF. This implies a continuous change of the quality of the chemical growth of calcium phosphates layers on substrates.

\subsection{Effects of SBF absorption on the flexural properties of PMMA composites}

Table 3 shows the flexural modulus and strength of PMMA and its composites after being subjected to SBF absorption for 1 and 2 months. Table 3 also shows the flexural properties (with their percentage of retention and percentage of recovery) of PMMA and its composites in wet and re-dried states after being subjected to SBF absorption. It can be seen that the flexural modulus and strength in wet states of PMMA/ $\gamma$-MPS treated 5HA composites was decreased after being subjected to SBF test for 1 month. The reduction of the flexural properties of

Table 3. Flexural properties of PMMA, PMMA/5HA composites and PMMA/ $\gamma$-MPS treated 5HA composites in wet and redried states after being subjected to SBF tests for various condition $(n=3)$

\begin{tabular}{|c|c|c|c|c|c|c|}
\hline \multirow{3}{*}{ Material } & \multirow{3}{*}{ Flexural properties } & \multirow{3}{*}{ Control } & \multicolumn{4}{|c|}{ Duration of immersion } \\
\hline & & & \multicolumn{2}{|c|}{1 month } & \multicolumn{2}{|c|}{2 months } \\
\hline & & & Wet & Redried & Wet & Redried \\
\hline \multirow{2}{*}{ PMMA } & Flexural modulus [GPa] & 2.3 & $2.4(103.6)$ & $2.7[117.1]$ & $2.4(105.0)$ & $2.5[110.8]$ \\
\hline & Flexural strength [MPa] & 77.7 & $82.9(106.7)$ & $90.9[117.0]$ & $80.8(103.9)$ & $88.1[113.4]$ \\
\hline \multirow{2}{*}{ PMMA/5HA } & Flexural modulus [GPa] & 2.5 & $2.4(95.9)$ & $2.7[107.4]$ & $2.5(98.5)$ & $2.5[99.3]$ \\
\hline & Flexural strength [MPa] & 50.5 & $57.9(114.8)$ & $73.0[144.6]$ & $64.0(126.8)$ & $62.7[124.2]$ \\
\hline \multirow{2}{*}{ PMMA/5HA treated $2 \% \mathrm{Si}$} & Flexural modulus [GPa] & 2.7 & $2.2(82.4)$ & $2.5[89.8]$ & $2.4(88.3)$ & 2.8 [104.1] \\
\hline & Flexural strength $[\mathrm{MPa}]$ & 56.5 & $42.7(80.6)$ & $53.9[102.2]$ & $44.1(83.6)$ & $57.2[107.7]$ \\
\hline \multirow{2}{*}{ PMMA/5HA treated $4 \% \mathrm{Si}$} & Flexural modulus [GPa] & 2.8 & $2.3(82.2)$ & $2.6[93.6]$ & $2.6(93.1)$ & $2.6[95.1]$ \\
\hline & Flexural strength [MPa] & 61.4 & $47.8(84.5)$ & $52.5[92.8]$ & $50.3(89.0)$ & $54.0[95.5]$ \\
\hline \multirow{2}{*}{ PMMA/5HA treated $6 \% \mathrm{Si}$} & Flexural modulus [GPa] & 2.9 & $2.3(79.9)$ & $2.7[93.8]$ & $2.6(89.4)$ & 2.9 [99.3] \\
\hline & Flexural strength $[\mathrm{MPa}]$ & 61.4 & $43.0(70.0)$ & $54.8[89.6]$ & $45.8(74.6)$ & $53.7[87.6]$ \\
\hline \multirow{2}{*}{ PMMA/5HA treated $8 \% \mathrm{Si}$} & Flexural modulus [GPa] & 2.8 & $2.4(86.3)$ & $2.6[94.1]$ & $2.6(92.5)$ & $2.9[102.4]$ \\
\hline & Flexural strength [MPa] & 52.4 & $46.7(89.2)$ & $56.5[107.9]$ & $45.8(87.4)$ & $59.3[113.2]$ \\
\hline
\end{tabular}

Remark 1: The values in parentheses ( ) are the percentage retention of the flexural properties after SBF absorption and the values in brackets [ ] are the percentage recovery of the flexural properties after re-drying

Remark 2: Statistically analyzed with one-way ANOVA correspond to various immersion condition indicate significant different $(P<0.05)$ in flexural modulus $\left(R^{2}=0.826\right)$. However, the flexural strength $\left(R^{2}=0.925\right)$ correspond to various immersion condition indicate no significant different $(P>0.05)$. 
these composites is due to the fact that the moisture contents may plasticize the composites, and thus weaken the bonding between HA filler and PMMA matrix due to the volumetric expansion within both phases. According to Sabbagh et al. [32], water molecules can lead to plasticizing effect in the matrix and cause degradation of the filler-matrix interface. The slightly reduction of the PMMA/ $\gamma$-MPS treated 5HA composites could be due to the undesirable and excessive loading of silane coupling agent. It is believed that the excess concentration of silane coupling agent (i.e. free and unreacted $\gamma$-MPS which located at the interface between the PMMA and HA) could contact with water molecule through hydrolytic reaction, and further cause the debonding of filler and matrix. In addition, the ion and water molecules in SBF can be accommodated at the interface between the filler and matrix

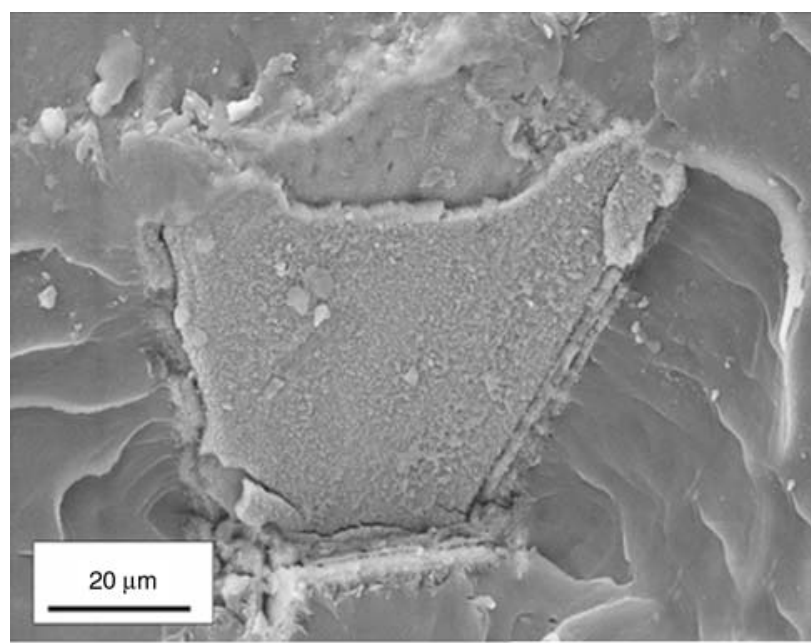

a)

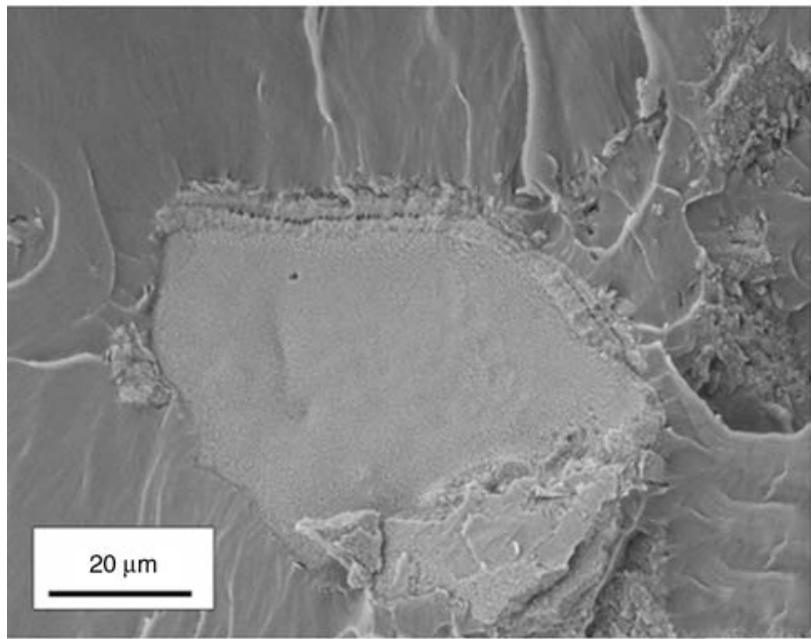

c) through a weak link. This weak link could provide paths of facile water molecule diffusion. According to Reis et al. [27], the increase and/or no significant change of flexural properties in denture base acrylic resin could be partically explained by continuous polymerization of the polymers. Residual monomer acts as plasticizer which by leaching out from the specimens could contribute to the increase in flexural properties after water immersion. On the contrary, water molecules absorbed during immersion may influence the mechanical properties of denture base acylic resins by the plasticizing effect. Table 3 shows that the flexural properties of the redried-PMMA/HA composites are higher than that of the wet-PMMA/HA composites after SBF tests. According to Cattani-Lorente et al. [33], water has two approach effects on resin-modified glass ionomer cement: (1) water acts can as a plasticizer

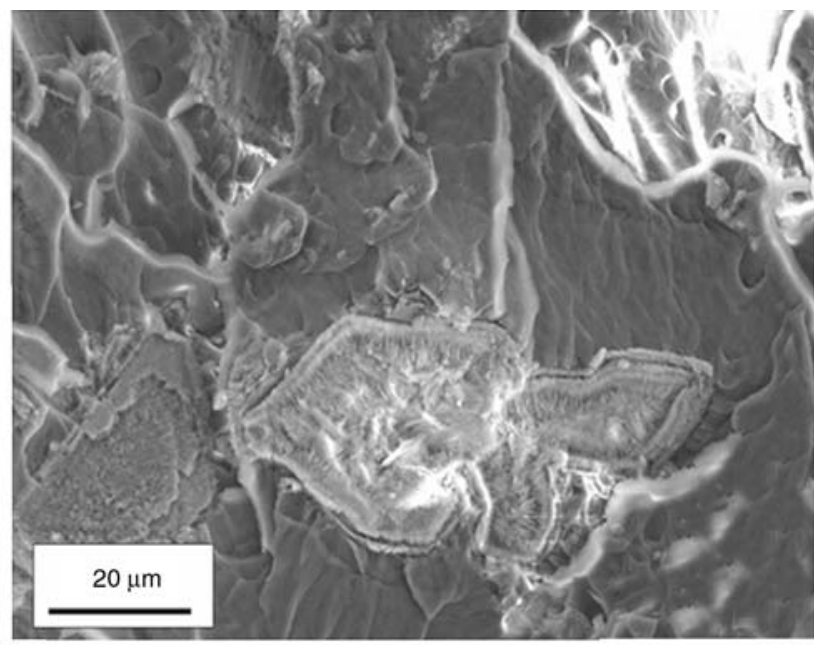

b)

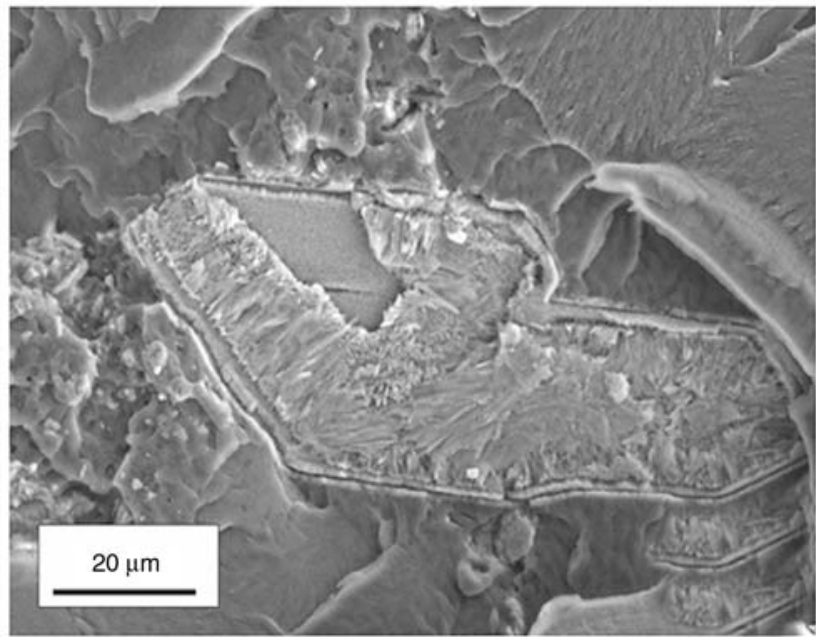

d)

Figure 4. FESEM micrographs taken from the fractured surfaces of (a) PMMA/5HA-control, (b) PMMA/5HA composites after being subjected to water absorption, (c) PMMA/ $\gamma$-MPS treated HA-control, and (d) PMMA/ $\gamma$-MPS treated HA composites after being subjected to water absorption 


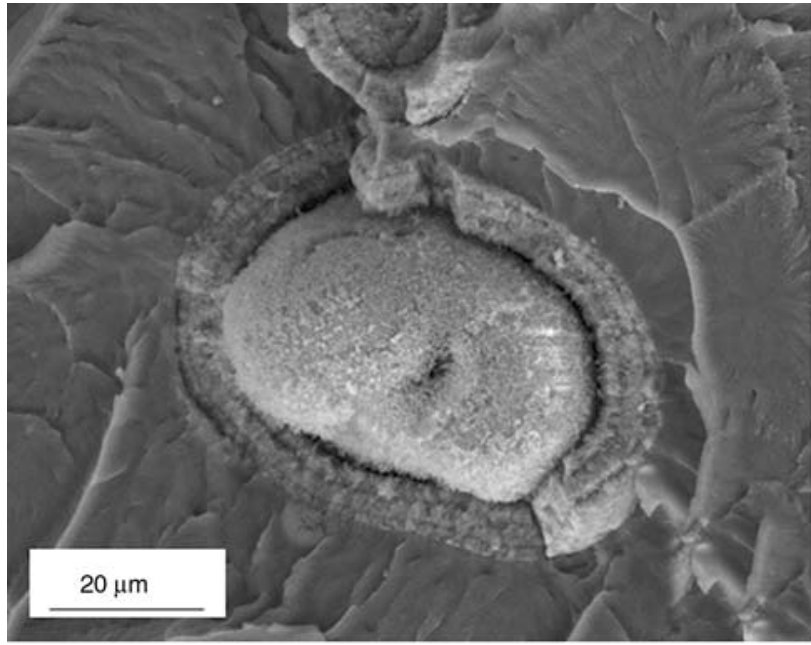

a)

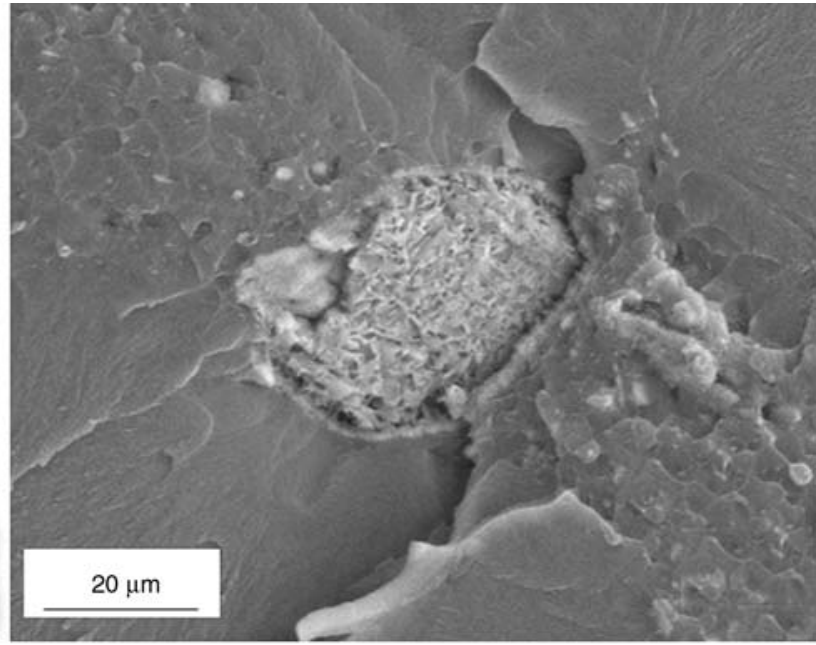

b)

Figure 5. FESEM micrographs taken from the fractured surfaces of (a) PMMA/5HA composites and (b) PMMA/ $\gamma$-MPS treated HA composites after being subjected to SBF immersion

and reduce the flexural strength. However, when excess water is removed, flexural strength of the samples almost reverted to initial values; (2) water also partly dissolved the components of the cement, changing the network structure, resulting in a slight but irreversible decrease of their strength. In addition, the presence of salts and proteins in the saliva will minimize the effect of water on the dental restorations.

\subsection{Morphological properties}

Figure 4 shows the FESEM micrographs taken from the fractured surfaces of PMMA/5HA and PMMA/ $\gamma$-MPS treated HA composites in their control state and after being subjected to water absorption. It can be seen that there is noticeable gap between PMMA and HA for the untreated PMMA/ 5HA composite (c.f. Figure 4a), however, for the PMMA $\gamma$-MPS treated HA composites, there is a better interfacial interaction between PMMA and HA (c.f. Figure 4c). From Figure 4b and 4d, it can be seen that the fracture morphology of the PMMA/ HA and PMMA $/ \gamma$-MPS treated HA composites did not change much. This fracture morphology can support the excellent recovery and retention properties of PMMA composites after being subjected to water absorption. Figure 5 shows the FESEM micrographs taken from the fractured surfaces of
PMMA/5HA composites and PMMA/ $\gamma$-MPS treated 5HA composites after being subjected to SBF immersion. Little de-bonding of the interfacial interaction between PMMA/5HA composites (with and without coupling agent) can be observed from the fractured surface. This may be related to the ions and water molecules in SBF that can be accommodated at the interface between the filler and matrix as a weak link.

\section{Conclusions}

The kinetics of water absorption and SBF absorption of PMMA/HA composites conformed to the Fickian Law behavior. It was found that the $\gamma$-MPS treated HA could help to reduce the water uptake due to the ability of $\gamma$-MPS to reacts with hydroxyl groups in the HA filler by introducing a hydrophobic layer on HA particles surface. The diffusion coefficient of PMMA/5HA composite (untreated and treated) is lower than that of PMMA. This is due to the present of HA particles which could restrict the diffusivity of water. The excellent retention and recovery of the PMMA/HA composites on mechanical properties could be attributed to the enhanced interfacial interaction between HA filler and PMMA matrix by the treatment of $\gamma$-MPS in water absorption. 


\section{Acknowledgements}

The authors would like to thanks MOSTI Malaysia for the Science Fund research grant, Universiti Sains Malaysia for the Research University Grant, Research University Postgraduate Research Grant Scheme and USM Fellowship. The authors would like to express their appreciation to Dr Sivakumar A/L Ramakrishnan (Universiti Sains Malaysia) for providing the technical knowledge in statistical analysis.

\section{References}

[1] Chen T., Kusy R. P.: Effect of methacrylic acid: Methyl methacrylate monomer ratios on polymerization rates and properties of polymethyl methacrylates. Journal of Biomaterials Materials Research Part A, 36, 190-199 (1997).

DOI: $10.1002 /($ SICI) 1097-4636(199708)36:2<190:: AID-JBM7>3.0.CO;2-F

[2] John J., Gangadhar S. A., Shah I.: Flexural strength of heat-polymerized polymethyl methacrylate denture resin reinforced with glass, aramid, or nylon fibers. The Journal of Prosthetic Dentistry, 86, 424-427 (2001). DOI: $10.1067 / \mathrm{mpr} .2001 .118564$

[3] Uzun G., Hersek N., Tinçer T.: Effect of five woven fiber reinforcements on the impact and transverse strength of a denture base resin. The Journal of Prosthetic Dentistry, 81, 616-620 (1999).

DOI: $10.1016 / \mathrm{S} 0022-3913(99) 70218-0$

[4] Kim S-H., Watts D. C.: The effect of reinforcement with woven E-glass fibers on the impact strength of complete dentures fabricated with high-impact acrylic resin. The Journal of Prosthetic Dentistry, 91, 274280 (2004).

DOI: $10.1016 /$ j.prosdent.2003.12.023

[5] Jagger D. C., Harrison A., Jandt K. D.: The reinforcement of dentures. Journal of Oral Rehabilitation, 26, 185-194 (1999).

DOI: $10.1046 / \mathrm{j} .1365-2842.1999 .00375 . \mathrm{X}$

[6] Hu Q. L., Li B. Q., Wang M., Shen J. C.: Preparation and characterization of biodegradable chitosan/hydroxyapatite nanocomposite rods via in situ hybridization: A potential material as internal fixation of bone fracture. Biomaterials, 25, 779-785 (2004). DOI: $10.1016 / \mathrm{S} 0142-9612(03) 00582-9$

[7] Morita S., Furuya K., Ishihara K., Nakabayashi N.: Performance of adhesive bone cement containing hydroxyapatite particles. Biomaterials, 19, 1601-1606 (1998).

DOI: $10.1016 / \mathrm{S} 0142-9612(97) 00120-8$

[8] Reeson M. G., Jepson N. J. A: Achieving an even thickness in heat-polymerized permanent acrylic resin denture bases for complete dentures. The Journal of Prosthetic Dentistry, 82, 359-361 (1999).

DOI: $\underline{10.1016 / \mathrm{S} 0022-3913(99) 70094-6}$
[9] Oysad H., Ruyter I. E.: Water sorption and filler characteristics of composites for use in posterior teeth. Journal of Dental Research, 65, 1315-1318 (1986). DOI: $10.1177 / 00220345860650110601$

[10] Deb S., Braden M., Bonfield W.: Water absorption characteristics of modified hydroxyapatite bone cements. Biomaterials, 16, 1095-1100 (995). DOI: $10.1016 / 0142-9612(95) 98906-\mathrm{U}$

[11] Drummond J. L.: Degradation, fatigue, and failure of resin dental composite materials. Journal of Dental Research, 87, 710-719 (2008). DOI: $\underline{10.1177 / 154405910808700802}$

[12] Dhanpal P., Yiu C. K. Y., King N. M., Tay F. R., Hirashi N.: Effect of temperature on water sorption and solubility of dental adhesive resins. Journal of Dentistry, 37, 122-132 (2009). DOI: $10.1016 / j . j d e n t .2008 .10 .004$

[13] Yiu C. K. Y., King N. M., Carrilho M. R. O., Sauro S., Rueggeberg F. A., Prati C., Carvalho R. M., Pashley D. H., Tay F. R.: Effect of resin hydrophilicity and temperature on water sorption of dental adhesive resin. Biomaterials, 27, 1695-1703 (2006).

DOI: 10.1016/j.biomaterials.2005.09.037

[14] Andrade Â. L., Turchetti-Maia R. M. M., Lopes M. T. P., Salas C. E., Domingues R. Z.: In vitro bioactivity and cytotoxicity of chemically treated glass fibers. Materials Research, 7, 635-638 (2004). DOI: $10.1590 /$ S1516-14392004000400019

[15] Palin W. M., Felming G. J. P., Burke F. J. T., Marquis P. M., Randall R. C.: The influence of short and medium-term water immersion on the hydrolytic stability of novel low-shrink dental composites. Dental Materials, 21, 852-863 (2005).

DOI: $10.1016 / j . d e n t a l .2005 .01 .004$

[16] Asaoka K., Hirano S.: Diffusion coefficient of water through dental composite resin. Biomaterials, 24, 975-979 (2003). DOI: $10.1016 / \mathrm{S} 0142-9612(02) 00435-0$

[17] Karabela M. M., Sideridou I. D.: Effect of the structure of silane coupling agent on sorption characteristics of solvents by dental resin-nanocomposites. Dental Materials, 24, 1631-1639 (2008). DOI: $10.1016 /$ j.dental.2008.02.021

[18] Liu Q., Ding J., Chambers D. E., Debnath S., Wunder S. L., Baran G. R.: Filler-coupling agent-matrix interactions in silica/polymethylmethacrylate composites. Journal of Biomedical Materials Research Part A, 57, 384-393 (2001).

DOI: $10.1002 / 1097-4636(20011205) 57: 3<384:: A I D-$ JBM1181>3.0.CO;2-F

[19] Söderholm K-J. M., Shang S-W.: Molecular orientation of silane at the surface of colloidal silica. Journal of Dental Research, 72, 1050-1054 (1993). DOI: $\underline{10.1177 / 00220345930720061001}$ 
[20] Beatty M. W., Swartz M. L., Moore B. K., Philips R. W., Roberts T. A.: Effect of microfiller fraction and silane treatment on resin composite properties. Journal of Biomedical Materials Research Part A, 40, 12-23 (1998).

DOI: 10.1002/(SICI) 1097-4636(199804)40:1<12:: AID-JBM2>3.0.CO;2-U

[21] Harper E. J., Braden M., Bonfield W.: Mechanical properties of hydroxyapatite reinforced poly(ethylmethacrylate) bone cement after immersion in a physiological solution: Influence of a silane coupling agent. Journal of Materials Science: Materials in Medicine, 11, 491-497 (2000).

DOI: $10.1023 / \mathrm{A}: 1013057724268$

[22] Yang H-S., Kim H-J., Park H-J., Lee B-J., Hwang TS.: Water absorption behavior and mechanical properties of lignocellulosic filler-polyolefin bio-composites. Composites Structures, 72, 429-437 (2006). DOI: 10.1016/j.compstruct.2005.01.013

[23] Santos C., Luklinska Z. B., Clarke R. L., Davy K. W. M.: Hydroxyapatite as a filler for dental composite materials: Mechanical properties and in vitro bioactivity of composites. Journal of Materials: Materials in Science, 12, 565-573 (2001). DOI: $10.1023 / \mathrm{A}: 1011291723503$

[24] Unemori M., Matsuya Y., Matsuya S., Akashi A., Akamie A.: Water absorption of poly(methyl methacrylate) containing 4-methacryloxyethyl trimellitic anhydride. Biomaterials, 24, 1381-1387 (2003). DOI: $10.1016 / \mathrm{S} 0142-9612(02) 00521-5$

[25] Tang Y. C., Chen D. Z., Yue T. M., Chan K. C., Tsui C. P., Peter Yu H. F.: Water absorption and solubility of PHBHV/HA nanocomposites. Composites Science and Technology, 68, 1927-1934 (2008). DOI: 10.1016/j.compscitech.2007.12.003

[26] Matinlinna J. P., Vallittu P. K.: Silane based concepts on bonding resin composite to metals. The Journal of Contemporary Dental Practice, 8, 1-8 (2007).
[27] Reis J. M. S. N., Vergani C. E., Pavarina A. C., Giampaolo E. T., Machado A. L.: Effect of relining, water storage and cyclic loading on the flexural strength of a denture base acrylic resin. Journal of Dentistry, 34, 420-426 (2006).

DOI: $10.1016 / \mathrm{j} . j d e n t .2005 .10 .001$

[28] Fan J. P., Tsui C. P., Tang C. Y., Chow C. L.: 3D finite element analysis of the damage effects on the dental composite subject to water sorption. Acta Mechanica Solida Sinica, 19, 212-222 (2006). DOI: 10.1007/s10338-006-0626-4

[29] Sousa R. A., Reis R. L., Cunha A. M., Bevis M. J.: Coupling of HDPE/hydroxyapatite composites by silane-based methodologies. Journal of Materials Science: Materials in Medicine, 14, 475-487 (2003). DOI: 10.1023/A:1023471011749

[30] Greish Y. E., Brown P. W.: Characterization of bioactive glass-reinforced HAp-polymer composites. Journal of Biomedical Materials Research Part A, 52, 687-694 (2000).

DOI: $10.1002 / 1097-4636(20001215) 52: 4<687::$ AIDJBM13>3.0.CO;2-K

[31] Verestiuc L., Morosanu C., Bercu M., Pasuk I., Mihailescu I. N.: Chemical growth of calcium phosphate layers on magnetron sputtered HA films. Journal of Crystal Growth, 264, 483-491 (2004). DOI: 10.1016/j.jcrysgro.2004.01.015

[32] Sabbagh J., Vreven J., Leloup G.: Dynamic and static moduli of elasticity of resin-based materials. Dental Materials, 18, 64-71 (2002). DOI: $10.1016 / \mathrm{S} 0109-5641(01) 00021-5$

[33] Cattani-Lorente M. A., Dupuis V., Payan J., Moya F., Meyer J-M.: Effect of water on the physical properties of resin-modified glass ionomer cement. Dental Materials, 15, 71-78 (1999). DOI: $10.1016 / \mathrm{S} 0109-5641(99) 00016-0$ 